\title{
Association of Cigarette Smoking with Hyperlipidemia in Male Individuals
}

\author{
Aminullah"1, Jahan Shah"1, Ali S. R. Alsubaie'2, Bismillah Sehar ${ }^{3}$, Farrukh Sher Khan', \\ Nawsherwan', Eltigani 0. M. Omer ${ }^{2}$, Alia Mohammed Almoajel5, Falak Zeb ${ }^{*}$
}

\begin{abstract}
${ }^{1}$ Department of Occupational Medicine and Environmental Health, School of Public Health, Nanjing Medical University, Nanjing, China

${ }^{2}$ College of Public Health, Imam Abdurhman Bin Faisal University, Dammam, Saudi Arabia

${ }^{3}$ Department of Public Health, National University of Medical Sciences, Islamabad, Pakistan

${ }^{4}$ Department of Preventive Medicine, School of Health Sciences, Wuhan University, Wuhan, China

${ }^{5}$ Department of Community Health Sciences, College of Applied Medical Sciences, King Saud University, Riyadh, Saudi Arabia

${ }^{6}$ Department of Human Nutrition and Dietetics, National University of Medical Sciences, Islamabad, Pakistan

Email: ^falak106@gmail.com
\end{abstract}

How to cite this paper: Aminullah, Shah, J., Alsubaie, A.S.R., Sehar, B., Khan, F.S., Nawsherwan, Omer, E.O.M., Almoajel, A.M. and Zeb, F. (2021) Association of Cigarette Smoking with Hyperlipidemia in Male Individuals. Food and Nutrition Sciences, 12, 937-949.

https://doi.org/10.4236/fns.2021.1210069

Received: August 29, 2021

Accepted: October 8, 2021

Published: October 11, 2021

Copyright $\odot 2021$ by author(s) and Scientific Research Publishing Inc. This work is licensed under the Creative Commons Attribution International License (CC BY 4.0).

http://creativecommons.org/licenses/by/4.0/ (c) (i) Open Access

\begin{abstract}
Cigarette smoking is one of the major modifiable and environmental risk factors which can alter the lipid profile that leads to the progression of atherosclerosis and cardiovascular diseases. The aim of the current study is to explore the association of cigarette smoking with Hyperlipidemia in male individuals. A cross-sectional study was carried out from March 2017 to August 2018 in Xuzhou, Jiangsu, China. A total of 1561 male individuals were enrolled in the study with a mean age (years) of $55.33 \pm 14.41$. We collected data on demographic, anthropometric and lifestyle indices. Total cholesterol (TC), triglyceride (TGL), and high-density lipoprotein cholesterol (HDL-C) were determined by the enzymatic colorimetric method. The mean level of serum TC, TG, and HDL-C were $4.85 \pm 0.91,1.69 \pm 1.45$ and $1.27 \pm 0.32 \mathrm{mmol} / \mathrm{L}$ respectively. We found that age, body mass index, pack-years, marital status, annual household income, alcohol consumption, smoking status, education level, and occupational status have significant association with Hyperlipidemia. Adjusted multiple logistic regressions showed that in old age, smoking behavior can significantly increase the risk of Hyperlipidemia. With an increase in pack-years, a significant increase is found only in TC while decreasing trend noticed in HDL-C level. Current smokers showed a significant increase in the risk of Hyperlipidemia compared to those who never smoked while smoking cessation decreases the risk of Hyperlipidemia. This study concluded that cigarette smoking along with increased age and pack-years can significantly increase the risk of Hyperlipidemia that further leads to heart diseases.
\end{abstract}




\section{Keywords}

Cigarette Smoking, Hyperlipidemia, Lipid Profile, Triglycerides

\section{Introduction}

Hyperlipidemia, a condition in which the level of lipids in the blood becomes abnormal, is characterized by the rise in total cholesterol (TC), low-density lipoprotein cholesterol (LDL-C) and triglyceride (TG) while a decrease in high-density lipoprotein cholesterol (HDL-C). Globally, Hyperlipidemia is more prevalent among adults. For instance, in the United States, about 53\% (105.3 million) of adults have lipid abnormalities [1]. Studies showed that the overall prevalence of Hyperlipidemia in China is $34.0 \%$, whereas $35.1 \%$ and $26.3 \%$ in urban and rural areas respectively [2]. Similar to changes in lifestyle, the prevalence of Hyperlipidemia is also increased over the recent decades in China [3]. A study from 2000 to 2001 by an International Collaborative Study of Cardiovascular Disease in Asia, reported that combined prevalence of borderline Hyperlipidemia and developed Hyperlipidemia in Chinese adults was 53.6\% (aged 35 - 74 years) [4]. In Chinese, the prevalence of Hyperlipidemia in men is significantly higher than women (41.9\% in men and $32.5 \%$ in women) [2]. Therefore, it is expected that the number of people with Hyperlipidemia will increase to 78 million in major countries by 2022, of which, one-sixth of the overall cases will happen in China [5] [6].

Hyperlipidemia is a major risk factor for cardiovascular disease (CVD) [7]. Globally, more than half of heart disease cases have been associated with Hyperlipidemia and more than 4 million deaths occur due to Hyperlipidemia every year [8]. As reported previously, high triglycerides and LDL-C are independently associated with the increased risk of coronary heart disease (CHD) [9] [10] [11]. Several prospective studies have concluded that the concentration of high-density lipoprotein cholesterol (HDL-C) is inversely associated with coronary heart disease. Therefore, the low concentration of HDL-C is a well-known risk factor for coronary heart disease [12]. Moreover, many epidemiological studies have shown that a few modifiable risk factors such as smoking are a major contributor to develop cardiovascular diseases (CVD).

China is one of the largest producers and consumers of tobacco in the world, which account for $38 \%$ of overall tobacco consumption [13], and around onethird of the world's cigarettes are consumed in China [14]. According to 2010 China Global Adults Tobacco Survey (GATS), 53\% of men at the age of 15 and above are current smokers [15]. The rate of smoking in males aged $\geq 40$ years is high in China [16]. Smoking can be triggered by many factors like age, race, gender and lifestyle etc. [17] [18]. Similar to developed countries, different studies in China suggested that individuals with a low educational level, income, and occupational status are more likely to smoke and less likely to quit smoking [19] 
[20] [21]. A pack-year is a clinical quantification of cigarette smoking used to measure a person's exposure to tobacco. This is used to assess their risk of developing diseases related to tobacco use. However, it is difficult to fully rely on the assessment based on the pack-year due to the different nature of the packaging by different companies [22].

Abnormal lipid profile has been associated with cigarette smoking. It is found that smoking-induced a rise in the level of TC, TG and decrease the level of HDL-C [23]. A study has shown that smoking elevates the level of TG and decreases the level of HDL-C in the blood of adult Chinese males and increases the risk of Hyperlipidemia [24]. Smoking had found a significant risk factor of many chronic diseases including cancer, lung diseases and CVD [25].

As previously mentioned, that the prevalence of smoking and Hyperlipidemia is high among Chinese adults. Most of the previous studies have focused on the combined association of smoking and alcohol consumption with lipid profile was seen in both genders. However, their individual association not clearly explored. Therefore, our study showed the association of cigarette smoking and pack years with Hyperlipidemia in male individuals in Xuzhou, Jiangsu-China.

\section{Material and Methods}

\subsection{Study Design and Participants}

We conducted a cross-sectional study from March 2017 to August 2018 in Xuzhou, in the north of Jiangsu province, China. A total of 3120 rural individuals were scrutinized out of which 1561 rural residents were eligible and finalized for the study through cluster random sampling from typical villages and towns of Tongshan district in Xuzhou. A total of 617 subjects who were combine smokers with alcohol consumption, 534 subjects who smoked occasionally, and 408 subjects who were females were excluded (Figure 1). The selected residents from the villages were mainly related to agriculture farming. The data was collected

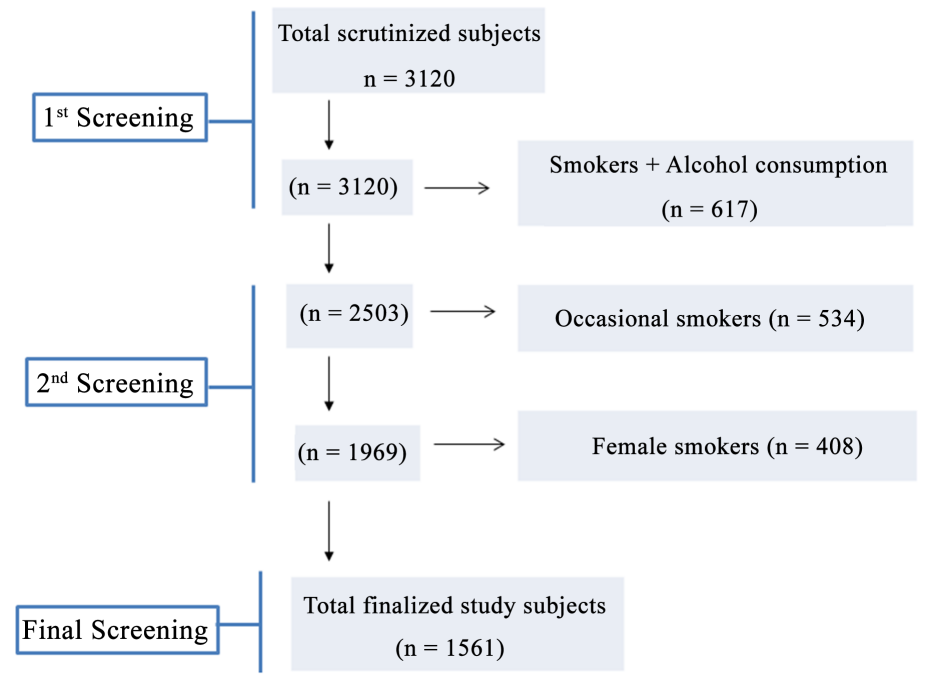

Figure 1. Flowchart of individual's recruitment. 
from the respondents after taken written consent form. Study participants were male individuals and were free of any chronic diseases such as CVD, kidney failure, liver disease, gastric problem, infectious diseases, and cancer etc., and were voluntarily agreed with the study protocol. The questionnaire and all the tools used in this study were in Chinese language. The questionnaire used for this study was newly constructed and validated through pilot testing after satisfactory translation into English language. The questionnaire was then kept confidential. The study was approved from the ethical board of Nanjing Medical University.

\subsection{Data Collection}

The study subjects were interviewed face to face by trained research assistants while using household questionnaires. Anthropometric, demographic and lifestyle data were collected and recorded in the questionnaire. Information about demographics was included age, income level, educational level, race, marital status, family and personal history. Lifestyle parameters were included smoking status and physical activity. We categorized smoking status into three groups, 1) Never smokers: Subjects who never smoke a cigarette in their life; 2) Ever smokers: Subjects who had quit smoking six months prior to the interview; 3) Current smokers: subjects who smoke on a daily basis for more than six months. The interviewers had filled and completed the questionnaires on the spot to avoid any miss information.

\subsection{Nutritional Status Assessment}

Anthropometric information was included BMI, waist circumference (WC), hip circumference (HC) and waist to hip ratio (WHR), calculated to determine the nutritional status of the participants according to WHO criteria. Weight and height of the individuals were measured by using a standard protocol. Individuals were instructed to remove footwear and heavy outfits. Weight was measured by a standard digital scale to the nearest $0.1 \mathrm{~kg}$. Height of the individuals was taken using an elastic measuring tape to the nearest $0.1 \mathrm{~cm}$. BMI was calculated from weight and height values by using the formula; $\mathrm{BMI}=$ weight in $\mathrm{kg} / \mathrm{height}$ in $\mathrm{m}^{2}$ [26] [27]. WC was measured around the navel point by using a flexible measuring tape on the horizontal plane in a standing position. HC was measured at the point at which circumference of the buttocks is maximum. Both measurements were recorded to the nearest $0.1 \mathrm{~cm}$. Waist to hip ratio was calculated by dividing the mean of waist circumference on the hip circumference.

\subsection{Serum Sample Collection}

A fasting $5 \mathrm{ml}$ blood was collected in EDTA tubes from the subjects and transferred to the laboratory of Occupational Medicine and Environmental Health, School of Public Health, Nanjing Medical University. Serum was separated by centrifugation for 10 minutes at $3000 \mathrm{rpm}$. The serum was then collected from the tubes and transferred to serum cups. The serum was kept at $-80^{\circ} \mathrm{C}$ for fur- 
ther analysis.

\subsection{Lipid Profile}

An automatic biochemical analyzer (HITACHI 7020, Japan) was used for the determination of lipid profile including TC, TG and HDL-C. Serum TC was determined by using the enzymatic colorimetric method. At first enzyme cholesterol esterase hydrolyzed the sample into cholesterol and fatty acids and then to hydrogen peroxide by enzyme cholesterol oxidase. The hydrogen peroxide then converted to red quinine in the presence of peroxidase enzyme. Then the intensity of the color was checked at $505 \mathrm{~nm}$ and correlated with the concentration of cholesterol in the sample. The method worked on the principle that is; triglycerides incubated together with lipoprotein lipase liberated glycerol and free fatty acids. In the presence of glycerol kinase and adenosine triphosphate, glycerol converted into glycerol-3-phosphate and adenosine-5-diphosphate. Glycerol-3phosphate then converted into dihydroxyacetone phosphate and hydrogen peroxide by glycerol phosphate dehydrogenase enzyme. In the presence of peroxidase, hydrogen peroxide reacts with 4 -aminophenazone and p-chlorophenol and gives a red color; the color intensity was measured at $505 \mathrm{~nm}$ for determination of triglyceride. HDL cholesterol-AUTO reagent, lipoprotein fractions separated by adding antibodies, which absorb into the surface of chylomicrons, VLDL and LDL. In the second step, detergent was added which broke HDL lipoproteins that make available the HDL cholesterol for quantitation by an enzymatic system. Dailab kits and reagents, Austria were used for lipid profile determination and recommended temperature was followed as per the experimental procedure by the manufacturer.

\subsection{Statistical Analysis}

We entered the data into SPSS 22.0 (SPSS Inc. Chicago, IL, USA) for statistical quantification. Descriptive statistics were measured by calculating the mean and standard deviation. Smoking risk factors and lipid profile levels were determined by applying chi-square test between the groups. Logistic regression was used to determine the smoking risk factors associated with Hyperlipidemia. A P value of less than 0.05 was considered statistically significant.

\section{Results}

\subsection{General Characteristics of the Study Population}

The mean age of the participants was $55.33 \pm 14.41$ while BMI was $24.33 \pm 4.61$. The percentage of non-smokers and smokers were $50.3 \%$ and $49.7 \%$ respectively. Among smokers, $7.4 \%$ of subjects were previous smokers and $42.3 \%$ current smokers. The mean of pack years for smokers was $33.57 \pm 14.73$. About half of the population $(58.3 \%$ ) was a normal lipid profile while $41.7 \%$ were recorded as hyperlipidemic. The mean of serum TC, TG, and HDL-C were $4.85 \pm 0.91,1.69$ \pm 1.45 and $1.27 \pm 0.32 \mathrm{mmol} / \mathrm{L}$ respectively (Table 1 ). 
Table 1. General characteristics of the study population $(n=1561)$.

\begin{tabular}{ccc}
\hline \multicolumn{2}{c}{ Variables } & Mean \pm S.D. (\%) \\
\hline Age (years) & BMI $\left(\mathrm{Kg} / \mathrm{m}^{2}\right)$ & $55.33 \pm 14.41$ \\
\hline Whether to smoke & No & $24.33 \pm 4.61$ \\
\hline Pack-years & Yes & $785(50.3 \%)$ \\
\hline \multirow{2}{*}{ Smoking status } & Never smoking & $776(49.7 \%)$ \\
\hline TC & Ever smoking & $33.57 \pm 14.73$ \\
\hline Th & Current smoking & $116(7.4 \%)$ \\
\hline Hyperlipidemia cases & No & $660(42.3 \%)$ \\
\hline HDL-C & Yes & $910(58.3 \%)$ \\
\hline
\end{tabular}

BMI $=$ Body mass index, Pack years $=$ Number of packs smoked per day ${ }^{*}$ years as a smoker, $\mathrm{TC}=$ Total cholesterol, TG $=$ Triglyceride, HDL-C $=$ High-density lipoprotein cholesterol.

\subsection{Screening of Risk Factors Associated with Hyperlipidemia}

The mean value of age, BMI and pack-years were $56.24 \pm 13.24$ and $58.17 \pm$ $14.18 ; 25.53 \pm 3.36$ and $23.48 \pm 5.16 ; 25.53 \pm 3.36$ and $23.48 \pm 5.16$ in cases and control groups respectively. The percentage of never, ever and current smoking status significantly changed between case and control group. There was a significant difference in the level of education, marital status, annual household income, alcohol consumption, and occupation status while no significant difference was found in physical activity, race and family history of Hyperlipidemia between two groups (Table 2).

\subsection{Correlation of Smoking with Risk of Hyperlipidemia in General Population on the Basis of Different Age Groups}

Logistic regression was used to analyze the relationship between smoking and the risk of Hyperlipidemia in general population of different age groups. The following models showed that an increase in age along with smoking behavior significantly increase the risk of high lipids in the blood. The risk of Hyperlipidemia was significantly higher in 45 - 60 and 60 - 75 years age groups than other age groups. This indicates that smoking can significantly increase the risk of Hyperlipidemia, especially in 45 - 60 and 60 - 75 years age groups (Table 3).

\subsection{Association of Smoking Years with Risk of Hyperlipidemia by Different Age Groups}

The study population was divided into a different age group. The number of years of smoking divided into 3 groups, for each group, the effects of smoking 
Table 2. Screening of risk factors associated with Hyperlipidemia.

\begin{tabular}{|c|c|c|c|c|c|}
\hline \multicolumn{2}{|c|}{ Variable } & $\begin{array}{l}\text { Controls } \\
(\mathrm{n}=910)\end{array}$ & $\begin{array}{c}\text { Cases } \\
(\mathrm{n}=651)\end{array}$ & $\lambda^{2}$ & $\mathbf{P}$ \\
\hline \multicolumn{2}{|c|}{ Age } & $58.17 \pm 14.18$ & $56.24 \pm 13.24$ & & 0.000 \\
\hline \multicolumn{2}{|c|}{ BMI } & $23.48 \pm 5.16$ & $25.53 \pm 3.36$ & & 0.000 \\
\hline \multicolumn{2}{|c|}{ Pack-years } & $23.48 \pm 5.16$ & $25.53 \pm 3.36$ & & 0.000 \\
\hline \multirow{2}{*}{$\begin{array}{l}\text { History of } \\
\text { smoking }\end{array}$} & No & $484(53.2 \%)$ & $301(46.2 \%)$ & \multirow{2}{*}{7.333} & \multirow{2}{*}{0.007} \\
\hline & Yes & $426(46.8 \%)$ & $350(53.8 \%)$ & & \\
\hline \multirow{3}{*}{ Smoking status } & Never & $484(53.2 \%)$ & $301(46.2 \%)$ & \multirow{3}{*}{7.337} & \multirow{3}{*}{0.026} \\
\hline & Ever & $64(7.0 \%)$ & $52(8.0 \%)$ & & \\
\hline & Current & $362(39.8 \%)$ & $298(45.8 \%)$ & & \\
\hline \multirow{4}{*}{ Race } & Han & $903(99.2 \%)$ & $642(98.6 \%)$ & \multirow{4}{*}{2.212} & \multirow{4}{*}{0.530} \\
\hline & Hui & $3(0.3 \%)$ & $5(0.8 \%)$ & & \\
\hline & Manchu & $3(0.3 \%)$ & $2(0.3 \%)$ & & \\
\hline & Other & $1(0.1 \%)$ & $2(0.3 \%)$ & & \\
\hline \multirow{5}{*}{$\begin{array}{l}\text { Education } \\
\text { status }\end{array}$} & Illiteracy & $169(18.6 \%)$ & $83(12.7 \%)$ & \multirow{5}{*}{18.195} & \multirow{5}{*}{0.001} \\
\hline & $\leq$ Primary & $267(29.3 \%)$ & $178(27.3 \%)$ & & \\
\hline & Secondary & $315(34.6 \%)$ & $242(37.2 \%)$ & & \\
\hline & High & $134(14.7 \%)$ & $112(17.2 \%)$ & & \\
\hline & $\geq$ High & $25(2.7 \%)$ & $36(5.5 \%)$ & & \\
\hline \multirow{3}{*}{$\begin{array}{l}\text { Marital } \\
\text { status }\end{array}$} & Not married & $48(5.3 \%)$ & $18(2.8 \%)$ & \multirow{3}{*}{8.671} & \multirow{3}{*}{0.013} \\
\hline & Married & $830(91.2 \%)$ & $619(95.1 \%)$ & & \\
\hline & Other & $32(3.5 \%)$ & $14(2.2 \%)$ & & \\
\hline \multirow{4}{*}{$\begin{array}{c}\text { Annual } \\
\text { household } \\
\text { income }\end{array}$} & $<3000$ & $185(20.3 \%)$ & $91(14 \%)$ & \multirow{4}{*}{18.674} & \multirow{4}{*}{0.000} \\
\hline & $3000-10,000$ & $404(44.4 \%)$ & $296(45.5 \%)$ & & \\
\hline & $10,000-30,000$ & $269(29.6 \%)$ & $198(30.4 \%)$ & & \\
\hline & $>30,000$ & $52(5.7 \%)$ & $66(10.1 \%)$ & & \\
\hline \multirow{3}{*}{$\begin{array}{c}\text { Alcohol } \\
\text { consumption }\end{array}$} & Non-drinks & $536(59.0 \%)$ & $369(56.8 \%)$ & \multirow{3}{*}{7.196} & \multirow{3}{*}{0.027} \\
\hline & Ever-drinkers & $28(3.1 \%)$ & $38(5.8 \%)$ & & \\
\hline & Drinkers & $345(38.0 \%)$ & $243(37.4 \%)$ & & \\
\hline \multirow{3}{*}{$\begin{array}{c}\text { Physical } \\
\text { activity }\end{array}$} & Frequently & $29(3.2 \%)$ & $23(3.6 \%)$ & & \\
\hline & Occasionally & $152(16.9 \%)$ & $124(19.2 \%)$ & 1.679 & 0.432 \\
\hline & Never & 721 (79.9\%) & $498(77.2 \%)$ & & \\
\hline & Farmers & $682(76 \%)$ & $467(73 \%)$ & & \\
\hline & Workers & $98(10.9 \%)$ & $44(6.9 \%)$ & & \\
\hline & TW & $29(3.2 \%)$ & $26(4.1 \%)$ & & \\
\hline Occupational & Drivers & $18(2 \%)$ & $16(2.5 \%)$ & 31.947 & 0.000 \\
\hline & Clerical workers & $42(4.7 \%)$ & $72(11.3 \%)$ & & \\
\hline & Retired staff & $20(2.2 \%)$ & $13(2 \%)$ & & \\
\hline & Students & $8(0.9 \%)$ & $2(0.3 \%)$ & & \\
\hline Family Hx of & No & $884(98.2 \%)$ & $631(97.8 \%)$ & 0.304 & 0.581 \\
\hline Hyperlipidemia & Yes & $16(1.8 \%)$ & $14(2.2 \%)$ & 0.504 & 0.301 \\
\hline
\end{tabular}

$\mathrm{BMI}=$ Body mass index, Pack years $=$ Number of packs smoked per day ${ }^{*}$ years as a smoker, $\mathrm{TW}=\mathrm{Tem}-$ porary workers. 
Table 3. Correlation of smoking with risk of Hyperlipidemia in general population on the basis of different age groups.

\begin{tabular}{|c|c|c|c|c|c|c|c|}
\hline & & \multicolumn{2}{|c|}{ Primary model } & \multicolumn{2}{|l|}{ Model I } & \multicolumn{2}{|l|}{ Model II } \\
\hline & & OR $(95 \% \mathrm{CI})$ & $\mathbf{P}$ & OR ( $95 \% \mathrm{CI})$ & $\mathbf{P}$ & OR $(95 \% \mathrm{CI})$ & $\mathbf{P}$ \\
\hline \multicolumn{2}{|c|}{ Total population $(\mathrm{N}=1561)$} & $1.32(1.08-1.62)$ & 0.007 & $1.45(1.17-1.79)$ & 0.001 & $1.55(1.24-1.93)$ & 0.000 \\
\hline \multirow{4}{*}{ Age groups } & $<45(\mathrm{n}=407)$ & $1.11(0.75-1.65)$ & 0.604 & $1.18(0.76-1.84)$ & 0.461 & $1.22(0.78-1.91)$ & 0.379 \\
\hline & $45-60(n=551)$ & $1.49(1.07-2.09)$ & 0.019 & $1.51(1.06-2.15)$ & 0.024 & $1.51(1.05-2.16)$ & 0.025 \\
\hline & $60-75(\mathrm{n}=477)$ & $1.21(0.84-1.76)$ & 0.307 & $1.79(1.17-2.71)$ & 0.007 & $1.15(1.09-1.22)$ & 0.000 \\
\hline & $>75(\mathrm{n}=126)$ & $2.00(0.87-4.58)$ & 0.103 & $2.49(0.99-6.29)$ & 0.054 & $1.84(1.21-2.81)$ & 0.005 \\
\hline
\end{tabular}

Initial model = No correction; Model I = Corrected by age, BMI, alcohol consumption, physical activity, family history of Hyperlipidemia. Model II = Corrected by age, BMI, WHI, culture, marital status, annual family income, alcohol consumption, occupation.

years on the risk of Hyperlipidemia were compared with the first fraction of smoking years, but no significant results were obtained. The effect of smoking years on the risk of Hyperlipidemia was not statistically significant (Table 4).

\subsection{Pack-Years Association with Lipid Profile According to Age Groups}

The relationship between pack-years and physical examination indices of Hyperlipidemia in different age groups were analyzed. Both the initial model and the corrected model showed that the longer the number of Pack-years in 45 - 60 years age group, the higher the cholesterol level of the subjects. The HDL-C values of the subjects in $>75$ years age group decreased with an increase in packyears, and the risk of Hyperlipidemia showed a significant trend (Table 5).

\section{Discussion}

This cross-sectional study opens many doors to further explore the association of smoking with Hyperlipidemia in different age groups in context of future perspective. As this study demonstrated by using physical observations and biochemical insight of 1561 individuals, that smoking is significantly associated with the lipid profile in different age groups. In addition, the present study reveals that there is a significant association of pack-years with total cholesterol, which is parallel with the results of a study by Xirofotos et al., observed that subjects with more pack-years can significantly increase the level of total cholesterol and reported a significant positive correlation between pack-years and total cholesterol [22]. Our study found that smoking can significantly increase the risk of Hyperlipidemia by bringing changes in blood lipids concentration especially in $45-60$ and 60 - 75 years age groups. This is in agreement with a study that was conducted in western China showed that in male subjects with an age of 65 years and above, smoking can significantly increase the risk of Hyperlipidemia [28].

The findings of the present study in term of serum TC $(4.85 \pm 0.91 \mathrm{mmol} / \mathrm{L})$, TG $(1.69 \pm 1.45 \mathrm{mmol} / \mathrm{L})$ and HDL-C $(1.27 \pm 0.32 \mathrm{mmol} / \mathrm{L})$ comparatively similar to a previous study, showed that the mean level of TC was $5.01 \pm 0.93$ $\mathrm{mmol} / \mathrm{L}, \mathrm{TG}(1.90 \pm 1.72 \mathrm{mmol} / \mathrm{L}), \mathrm{HDL}-\mathrm{C}(1.21 \pm 0.35 \mathrm{mmol} / \mathrm{L})$ and LDL-C 
Table 4. Association of smoking years with risk of Hyperlipidemia by different age groups.

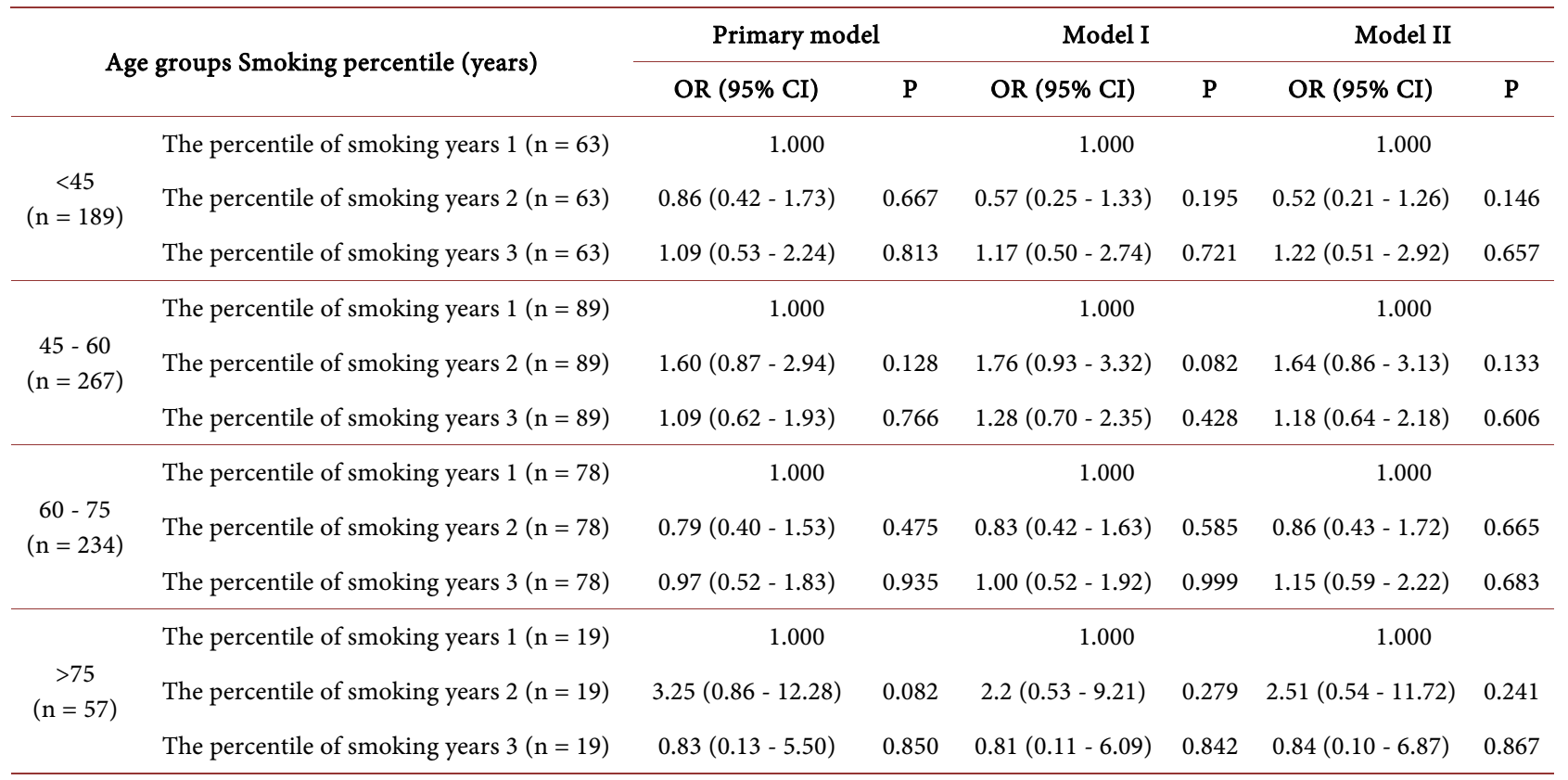

Initial model = No correction; Model I = Corrected by age, BMI, alcohol consumption, physical activity, family history of Hyperlipidemia. Model II = Corrected by age, BMI, WHI, culture, marital status, annual family income, alcohol consumption, occupation.

Table 5. Pack-years association with lipid profile according to age groups.

\begin{tabular}{|c|c|c|c|c|c|c|c|}
\hline & & \multicolumn{2}{|c|}{ Primary model } & \multicolumn{2}{|l|}{ Model I } & \multicolumn{2}{|l|}{ Model II } \\
\hline & & OR (95\% CI) & $\mathbf{P}$ & OR (95\% CI) & $\mathbf{P}$ & OR (95\% CI) & $P$ \\
\hline & TC & $0.74(0.40-1.87)$ & 0.202 & $0.57(0.66-1.80)$ & 0.363 & $0.39(0.77-1.56)$ & 0.507 \\
\hline \multirow[t]{3}{*}{$<45(\mathrm{n}=189)$} & TG & $0.08(0.76-0.59)$ & 0.810 & $0.25(0.98-0.47)$ & 0.491 & $0.26(0.96-0.44)$ & 0.467 \\
\hline & HDL-C & $3.24(-0.69-7.17)$ & 0.105 & $4.28(-0.01-8.58)$ & 0.051 & $3.27(-0.71-7.25)$ & 0.106 \\
\hline & $\mathrm{TC}$ & $1.29(2.27-0.31)$ & 0.010 & $1.26(2.26-0.25)$ & 0.014 & $1.13(2.14-0.12)$ & 0.028 \\
\hline \multirow[t]{3}{*}{$45-60(n=267)$} & TG & $0.03(0.56-0.62)$ & 0.917 & $0.07(0.57-0.71)$ & 0.825 & $0.27(0.37-0.91)$ & 0.411 \\
\hline & HDL-C & $-1.84(-4.52-0.85)$ & 0.179 & $-2.05(-5.03-0.93)$ & 0.176 & $-2.82(-5.80-0.16)$ & 0.064 \\
\hline & $\mathrm{TC}$ & $0.27(1.51-0.97)$ & 0.669 & $0.09(-1.37-1.19)$ & 0.890 & $0.08(1.36-1.19)$ & 0.897 \\
\hline \multirow[t]{3}{*}{$60-75(\mathrm{n}=234)$} & TG & $0.51(1.49-0.46)$ & 0.303 & $0.29(-1.31-0.74)$ & 0.581 & $0.29(1.30-0.71)$ & 0.564 \\
\hline & HDL-C & $-0.28(-3.91-3.35)$ & 0.880 & $-1.76(-5.65-2.14)$ & 0.375 & $-0.73(-4.62-3.16)$ & 0.711 \\
\hline & TC & $1.23(3.14-0.67)$ & 0.200 & $1.53(3.55-0.49)$ & 0.135 & $1.23(3.72-1.26)$ & 0.325 \\
\hline \multirow[t]{2}{*}{$>75(\mathrm{n}=57)$} & TG & $0.29(1.95-1.37)$ & 0.727 & $0.35(2.39-1.69)$ & 0.732 & $0.01(2.12-2.15)$ & 0.992 \\
\hline & HDL-C & $-4.30(-8.71-0.1)$ & 0.055 & $-6.18(-11.16-1.21)$ & 0.016 & $-5.96(-11.18-0.75)$ & 0.026 \\
\hline
\end{tabular}

$\mathrm{TC}=$ Total cholesterol, $\mathrm{TG}=$ Triglyceride, HDL-C = High-density lipoprotein cholesterol; Initial model = No correction; Model I = Corrected by age, BMI, alcohol consumption, physical activity, family history of Hyperlipidemia. Model II = Corrected by age, BMI, WHI, culture, marital status, annual family income, alcohol consumption, occupation.

$(3.15 \pm 0.80 \mathrm{mmol} / \mathrm{L})$ [3]. A population-based cohort study reported that age, male, occupation, type of work, marital status, duration of service, family income, education levels, smoking status, smoking index, alcohol consumption, BMI, and obesity were significantly related to Hyperlipidemia [29] [30]. In line with 
these results, our study demonstrates that age, BMI, marital status, family income, alcohol consumption, smoking status, pack-years, education level, and occupation are significant risk factors of Hyperlipidemia.

A cross-sectional study that was carried out from January 2013 to September 2013 in Sudanese male population, found that there was an increased trend noted in the mean level of TC and a declining trend had been observed in HDL-C level with increase in the duration of smoking, while no effect has been observed in serum TG level [31]. Withstand with these findings the current study also reported that, increased smoking years can significantly increase the level of TC and decrease HDL-C level without bringing any change in TG values of the individuals.

A large meta-analysis showed that the level of serum TC, TG, VLDL, and LDL were significantly higher with a low concentration of HDL-C in smoker subjects as compared to non-smoker subjects [24]. It means that the subjects who had been smoking increase their risk of Hyperlipidemia as compared to those who never smoked. Similarly, another meta-analysis concluded that cigarette smoking cessation increases the level of HDL-C without affecting the levels of TC, LDL-C, and TG [32]. It reflected that subjects who quit smoking decrease the risk of developing Hyperlipidemia. In consistent with the results of these meta-analyses the current study also reported that the risk of Hyperlipidemia increased significantly in subjects who had been smoking compared to those who never smoked. While there was no association found between the history of smoking cessation with the risk of Hyperlipidemia. Increment in HDL-C level after quitting cigarette smoking is reported beneficial for health and indicated that subjects who quit smoking decrease the risk of developing Hyperlipidemia. In addition, a recent study demonstrated that smoking has a negative influence on some aspects of lifestyle including eating habits, exercise and sleeping pattern that contribute to disturb the rhythm of metabolism and life [33].

This study has some potential limitation. As the design of the study was crosssectional by which we cannot predict the exact causality. Second, we used questionnaires which is a retrospective method of survey, therefore there was recalling errors since it depends on subject's recalling memory that could possibly result in overestimation or underestimation of the observed association of smoking with lipid profile. More prospective and observational studies are needed to explore the causal effect of smoking on the lipid profile of smokers. Temporarily, there is a need for collection and quantification of more complete data about smoking cessation. Moreover, dietary behavior and physical workout are the most important factors for a lipid profile that need to be considered in future studies. As our study is observational the sample size is not much enough. Previous studies indicated that the effects of smoking on lipid profile need quiet enough time to be detected correctly, which need for more follow-up studies with big sample size to explore the time effect and association of smoking with Hyperlipidemia. 


\section{Conclusion}

Our study concluded that cigarette smoking along with increased age and pack years is associated with Hyperlipidemia in male individuals. Intervention strategies are needed to prevent Hyperlipidemia and control risk factors for cardiovascular disease.

\section{Conflicts of Interest}

The authors declare no conflict of interest regarding this paper.

\section{Funding Source}

There was no external funding for this study.

\section{References}

[1] Toth, P.P., Potter, D. and Ming, E.E. (2012) Prevalence of Lipid Abnormalities in the United States: The National Health and Nutrition Examination Survey 20032006. Journal of Clinical Lipidology, 6, 325-330. https://doi.org/10.1016/j.jacl.2012.05.002

[2] Pan, L., Yang, Z., Wu, Y., Yin, R.X., Liao, Y., Wang, J., et al. (2016) The Prevalence, Awareness, Treatment and Control of Dyslipidemia among Adults in China. Atherosclerosis, 248, 2-9. https://doi.org/10.1016/j.atherosclerosis.2016.02.006

[3] Wang, S., Xu, L., Jonas, J.B., You, Q.S., Wang, Y.X. and Yang, H. (2011) Prevalence and Associated Factors of Dyslipidemia in the Adult Chinese Population. PLoS ONE, 6, e17326. https://doi.org/10.1371/journal.pone.0017326

[4] Gu, D., Gupta, A., Muntner, P., Hu, S., Duan, X., Chen, J., et al. (2005) Prevalence of Cardiovascular Disease Risk Factor Clustering among the Adult Population of China: Results from the International Collaborative Study of Cardiovascular Disease in Asia (InterAsia). Circulation, 112, 658-665. https://doi.org/10.1161/CIRCULATIONAHA.104.515072

[5] Comanor, W.S. and Scherer, F.M. (2013) Mergers and Innovation in the Pharmaceutical Industry. Journal of Health Economics, 32, 106-113.

https://doi.org/10.1016/j.jhealeco.2012.09.006

[6] Zhao, W.H., Zhang, J., Zhai, Y., You, Y., Man, Q.Q., Wang, C.R., et al. (2007) Blood Lipid Profile and Prevalence of Dyslipidemia in Chinese Adults. Biomedical and Environmental Sciences, 20, 329-335.

[7] Kopin, L. and Lowenstein, C. (2017) Dyslipidemia. Annals of Internal Medicine, 167, ITC81-ITC96. https://doi.org/10.7326/AITC201712050

[8] Smith, D.G. (2007) Epidemiology of Dyslipidemia and Economic Burden on the Healthcare System. The American Journal of Managed Care, 13, S68-S71.

[9] Di Angelantonio, E., Sarwar, N., Perry, P., Kaptoge, S., Ray, K.K., et al. (2009) Major Lipids, Apolipoproteins, and Risk of Vascular Disease. JAMA, 302, 1993-2000. https://doi.org/10.1001/jama.2009.1619

[10] Schlant, R.C., Forman, S., Stamler, J. and Canner, P.L. (1982) The Natural History of Coronary Heart Disease: Prognostic Factors after Recovery from Myocardial Infarction in 2789 Men. The 5-Year Findings of the Coronary Drug Project. Circulation, 66, 401-414. https://doi.org/10.1161/01.CIR.66.2.401

[11] Pekkanen, J., Linn, S., Heiss, G., Suchindran, C.M., Leon, A., Rifkind, B.M., et al. 
(1990) Ten-Year Mortality from Cardiovascular Disease in Relation to Cholesterol Level among Men with and without Preexisting Cardiovascular Disease. The New England Journal of Medicine, 322, 1700-1707. https://doi.org/10.1056/NEJM199006143222403

[12] Billinger, S., Arena, R. and Bernhardt, J. (2014) A Statement for Healthcare Professionals from the American Heart Association/American Stroke Association. Stroke, 45, 2532-2253. https://doi.org/10.1161/STR.0000000000000022

[13] Eriksen, M., Mackay, J. and Ross, H. (2013) The Tobacco Atlas. American Cancer Society.

[14] Yang, G. (2014) Marketing 'Less Harmful, Low-Tar' Cigarettes is a Key Strategy of the Industry to Counter Tobacco Control in China. Tobacco Control, 23, 167-172. https://doi.org/10.1136/tobaccocontrol-2012-050691

[15] Yang, G.H., Li, Q., Wang, C.X., Hsia, J., Yang, Y., Xiao, L., et al. (2010) Findings from 2010 Global Adult Tobacco Survey: Implementation of MPOWER Policy in China. Biomedical and Environmental Sciences, 23, 422-429. https://doi.org/10.1016/S0895-3988(11)60002-0

[16] Wang, N., Feng, Y.J., Bao, H.L., Cong, S., Fan, J., Wang, B.H., et al. (2018) Survey of Smoking Prevalence in Adults Aged 40 Years and Older in China, 2014. Chinese Journal of Epidemiology, 39, 551-556.

[17] Mistry, R., McCarthy, W.J., de Vogli, R., Crespi, C.M., Wu, Q. and Patel, M. (2011) Adolescent Smoking Risk Increases with Wider Income Gaps between Rich and Poor. Health \& Place, 17, 222-229. https://doi.org/10.1016/j.healthplace.2010.10.004

[18] Mackenbach, J.P., Stirbu, I., Roskam, A.J., Schaap, M.M., Menvielle, G., Leinsalu, M., et al. (2008) Socioeconomic Inequalities in Health in 22 European Countries. The New England Journal of Medicine, 358, 2468-2481. https://doi.org/10.1056/NEJMsa0707519

[19] Pan, Z. (2004) Socioeconomic Predictors of Smoking and Smoking Frequency in Urban China: Evidence of Smoking as a Social Function. Health Promotion International, 19, 309-315. https://doi.org/10.1093/heapro/dah304

[20] Le, C., Chongsuvivatwong, V., Geater, A. and Apakupakul, N. (2009) Contextual and Individual Demographic Determinants of Alcohol Consumption and Smoking: A Comparative Study in Southwestern China and Southern Thailand. The Southeast Asian Journal of Tropical Medicine and Public Health, 40, 370-379.

[21] Kenkel, D., Lillard, D.R. and Liu, F. (2009) An Analysis of Life-Course Smoking Behavior in China. Health Economics, 18, S147-S156.

https://doi.org/10.1002/hec.1507

[22] Xirofotos, D., Trakakis, E., Peppa, M., Chrelias, C., Panagopoulos, P., Christodoulaki, C., et al. (2016) The Amount and Duration of Smoking is Associated with Aggravation of Hormone and Biochemical Profile in Women with PCOS. Gynecological Endocrinology, 32, 143-146. https://doi.org/10.3109/09513590.2015.1101440

[23] Okamura, T. (2010) Dyslipidemia and Cardiovascular Disease: A Series of Epidemiologic Studies in Japanese Populations. Journal of Epidemiology, 20, 259-265. https://doi.org/10.2188/jea.JE20100060

[24] Craig, W.Y., Palomaki, G.E. and Haddow, J.E. (1989) Cigarette Smoking and Serum Lipid and Lipoprotein Concentrations: An Analysis of Published Data. British Medical Journal, 298, 784-788. https://doi.org/10.1136/bmj.298.6676.784

[25] Jia, W.P. (2013) The Impact of Cigarette Smoking on Metabolic Syndrome. Biomedical and Environmental Sciences, 26, 947-952. 
[26] Guilbert, J.J. (2003) The World Health Report 2002: Reducing Risks, Promoting Healthy Life. Education for Health, 16, Article No. 230.

[27] WWH (2004) Reducing Risks, Promoting Healthy Life. Geneva.

[28] Tan, X.J., Jiao, G.P., Ren, Y.J., Gao, X.R., Ding, Y., Wang, X.R., et al. (2008) Relationship between Smoking and Dyslipidemia in Western Chinese Elderly Males. Journal of Clinical Laboratory Analysis, 22, 159-163. https://doi.org/10.1002/jcla.20235

[29] Su, M., Fu, C., Li, S., Ying, X., He, N. and Jiang, Q. (2013) Prevalence of Hyperlipidemia and Possible Risk Factors in Rural Chinese Adults: Cohort Study of Health Population in Yuhuan Rural. Journal of Hygiene Research, 42, 724-729.

[30] Fan, Y., Huang, J.J., Sun, C.M., Qiao, N., Zhang, H.X., Wang, H., et al. (2017) Prevalence of Dyslipidaemia and Risk Factors in Chinese Coal Miners: A Cross-Sectional Survey Study. Lipids in Health and Disease, 16, Article No. 161. https://doi.org/10.1186/s12944-017-0548-9

[31] Hassan, E.E., Gabra, H.M., Abdalla, Z.A. and Ali, A.E. (2013) Effect of Cigarette Smoking on Lipid Profile in Male at Collage of Police and Low Khartoum, Sudan. Asian Journal of Biomedical and Pharmaceutical Sciences, 3, 28-31.

[32] Maeda, K., Noguchi, Y. and Fukui, T. (2003) The Effects of Cessation from Cigarette Smoking on the Lipid and Lipoprotein Profiles: A Meta-Analysis. Preventive Medicine, 37, 283-290. https://doi.org/10.1016/S0091-7435(03)00110-5

[33] Omasu, F., Komori, A., Higashi, K. and Yoshimura, H. (2021) Impact of Smoking on Lifestyle and Vitality in College Students. Open Journal of Preventive Medicine, 11, 199-210. https://doi.org/10.4236/ojpm.2021.115016 